Volume 8, No.4, July - August 2019

International Journal of Advanced Trends in Computer Science and Engineering

Available Online at http://www.warse.org/IJATCSE/static/pdf/file/ijatcse105842019.pdf

https://doi.org/10.30534/ijatcse/2019/105842019

\title{
An Enriched Chaotic Quantum Whale Optimization Algorithm Based Job scheduling in Cloud Computing Environment
}

\author{
G. Kiruthiga ${ }^{1}$, Dr. S. Mary Vennila ${ }^{2}$ \\ PG \& Research Department of Computer Science, Presidency College, Chennai, India, g_kiruthiga @ yahoo.co.in ${ }^{1}$ \\ PG \& Research Department of Computer Science, Presidency College, Chennai, India, \\ vennilahymend@yahoo.co.in ${ }^{2}$
}

\begin{abstract}
In recent years, cloud has become a metaphor for voluminous data storage and utilization of virtual resources by cloud user. This study focuses on independent job scheduling in cloud computing paradigm. This paper devised a new enriched approach of chaotic quantum whale optimization algorithm (CQWOA), whose ultimate objective is to overwhelm degree of imbalance, increasing makespan and overheads in cost, energy consumption, resource utilization. With the intelligence of chaotic mapping and the quantum mechanism based optimal virtual machine selection, the global optima is achieved more significantly by CQWOA. This algorithm discovers best location and the direction to detect appropriate virtual machine in terms of reduced resource utilization, increasing makespan and evenly distributing the work load, makes the presented model to be more superior than Particle swarm optimization, Ant colony Optimization and standard Whale optimization. The existing models fails to handle the inconsistencies and vagueness in discovering potential virtual machine's which qualifies their requirements and standard whale optimization easily meets earlier converge of local optima and it is very complex for them to reach global best virtual machines in cloud computing Paradigm. The proposed CQWOA model has saved the total execution cost in job scheduling more successfully and it is proved by its simulation results.
\end{abstract}

Key words: Job scheduling, significant, chaotic mapping, quantum mechanism, whale optimization, local optima, global optima, cloud computing.

\section{INTRODUCTION}

Amid of all fiercely competitive technologies emerging in IT field, cloud computing is one of the most significant fields. Approximately each company pushing its infrastructure into cloud empowering system which intensifies the increase in productivity as well as to afford more continuous services with qualified comfort. The main awareness about cloud computing is that it permits users to store their data remotely on third party data centers and access it from anywhere an internet connection is available [1]. With its reliability now cloud computing is highly accepted paradigm for its high performance by providing many IT functionalities to the users as service. The service providers are termed as cloud in cloud computing, they hold all kinds of computing, storage resources, etc.

They chiefly provide three levels of services like Infrastructure as a Service (IaaS) which provides virtualized hardware and software, Platform as a Service (PaaS) which provides user to deploy their web applications and reduces the complexity in using high level programming and Software as a Service (SaaS) in which service providers develop software applications and host on cloud, the user can subscribe and start using that application without worrying about updating of software or purchasing hardware [2].

Apart from this, one of the main activities done by cloud computing paradigm is job scheduling. To increase the efficiency of cloud computing, managing the scheduling process is very important task. The main objective of job scheduler in cloud computing is sharing the load on processors in a balanced manner, to maximize the resource utilization and minimize the overall execution time of each job. But handling job scheduling is the toughest challenge in cloud computing, because it works under shared environment. Several algorithms are developed to handle the job scheduling process based on the number of resources available and the user's requirements. It is easy to schedule job if they are simple, but it not in case of complex applications in which it requires more complex and efficient scheduling scheme.

This paper focusses on developing a novel bio inspirational meta heuristic method for scheduling jobs in cloud computing with the intelligence of whale's behaviour.

\section{RELATED WORK}

Salim Bitam [3] developed a novel bee swarm optimization to schedule the jobs with available resources in cloud data centers. Its performance is compared with the genetic algorithm. The results show that bee life algorithm generates less execution time comparing with genetic algorithm with less complexity. Arash Ghorbannia et al [4] in their work introduced a consistent scheduling scheme in cloud computing paradigm. In this model, each main job is 
partitioned into sub jobs, to balance the jobs. The request time and response time are computed separately. Each job is scheduled on the basis of request and response time computed based on shared job. So that the competence of the model is increased.

Verma \& Kaushal [5] presented a scheduling scheme which assigns the job is such a way to reduce the cost of executing and facing the time limitations opted by the users, in an efficient way. Wang and Ai [6] devised a policy-oriented task scheduling using Ant Colony Optimization (ACO). In this work some aspect of ACO was modified to solve the problem of task scheduling in cloud computing environment. This was achieved by increasing the datacenter broker class to design and develop a new ACO based task scheduling scheme. Makespan is used to evaluate the performance of the standard policy and proposed scheduling policy. From the experimental results it is revealed that the ACO based task scheduling scheme decreases the make span of the task very effectively.

Demyana Izzat Esa and Adil Yousif [ 7] introduced a novel firefly-based job scheduling algorithm which minimize the job execution. Based on the collection information about job length, resource capacity and identifiers. Initially the jobs are assigned to the resources in an arbitrary fashion, and computes the fitness function which discovers the jobs execution time. Next, depending on the firefly behavior the populations are generated during each iteration, to produce best job scheduling scheme which minimize the overall jobs execution time.

Jun Xue et al [8] produced a genetic hybrid particle swarm optimization to enhance the quality of the service during job scheduling. The crossover, mutation are the operations of genetic algorithm is adapted in particle swarm optimization. The performance analysis revealed that GHPSO produces better result while comparing with conventional PSO in terms of minimizing execution time.

Guo Zhao et al [9] devised a task scheduling method by adapting PSO method which relies on change of less position value to reduce the cost minimization. PSO algorithm wile embedded with genetic operations its convergence rate is decrease but it executes faster than other two algorithms. The modified PSO method is more appropriate to suit job scheduling in cloud environment.

Pandey et al [10] used particle swarm optimization which handles the problem of scheduling in a heuristic manner in cloud resource management. By varying its communication and computation cost are used for determining the workflow application. The results expose that the cost savings and workload distribution is even.

Varalakshmi et al [11] discovers a solution which meets the quality of service with improvement in CPU utilization. The job scheduling scheme depends on the cloud workflow and the availability of resources.

Zhong et al [12] developed an improved genetic algorithm which involves in scheduling algorithm, which attains the optimization in cloud environment. This process performs automated policy of scheduling. The speed for improved genetic algorithm is double times of standard GA and utilizes the resources with higher rate.

Selvarani et al [13] performed the measure on both computation and execution cost of resource utilization. The cost scheduling method makes assignment of jobs based on the availability of resources in cloud paradigm. This model enhances the communication rate by gathering the task of users corresponding to a specific cloud resources based on their capability and assign the gathered jobs to corresponding resource.

Karnam and Sreelatha [19] in their work introduced $\mathrm{W}$-scheduler based task scheduling scheme which is computed based on fitness value by considering budget cost and their makespan. Nasiri \& Khiyabani [20] developed a novel meta-heuristic clustering approach which uses Whale Clustering Optimization Algorithm, constructed on the swarm foraging behavior of humpback whales.

Madhuravani et al [21] in their work developed a elementary security assurance model, in which the information passed over the cloud is encoded using RSA with MD5 and it is authenticated for validating the sender they used Diffie-Hellman computation, which is propelled in the cloud, hence this method guarantees security and privacy of data. Muhammad et al [22] addresses the issue of data leakage during virtual machine migration and authentication in cloud computing paradigm. To overcome this issue, they proposed packet capturing model which determines the data leakage, which efficiently to discover the presence of data leakage, during the traffic is not encrypted in cloud computing.

This paper work aims at developing a job scheduling in cloud environment by adapting the chaos quantum whale optimization for handling the problems of earlier convergence to local optima and enhanced the process of execution time to minimize the make span, in which the existing nature -inspired metaheuristic algorithm fails to achieve.

\section{BEHAVIOUR OF WHALE OPTIMIZATION ALGORITHM}

Whale optimization Algorithm (WOA) is an optimized meta-heuristic model developed based on the inspiration of humpback whales hunting behavior [14]. The humpback whales mainly have small fish herds or krill near the sea surface. The humpback whales, once discovered their prey they go to the bottom of the prey about 12 meters and then generate bubbles along a 9 shaped path. Meanwhile, whales upstream towards the sea surface, over the bubbles to enclose the prey. This algorithm involves in three main process of hunting they are encircling prey, probing for prey and attacking the prey. This algorithm best suits for resource and job scheduling in distributed system [15]. 


\subsection{Encircling prey}

After conforming the location of prey, the humpback whales start to encircle their prey. As the position of prey is unknown in real time, WOA adopt the current optimal solution is prey position or else the position close to the optimal solution. Meanwhile, other individuals involve in searching updates their position information towards present optimal solution. The encircling of prey can be signified as follows:

$$
\begin{aligned}
& E=|D . \hat{Y}(t)-Y(t)| \\
& Y(t+1)=\hat{Y}(t)-B . E \\
& B=2 a \cdot r-a \\
& D=2 r
\end{aligned}
$$

Where $t$ denotes the current iteration, $\mathrm{B}$ and $\mathrm{D}$ defines coefficient vectors, E represents distance among current optimal individual $\hat{Y}(t)$ and current individual $Y(t)$ at $t^{\text {th }}$ iteration, $\hat{Y}(t)$ defines the position vector of the optimal individual achieved so for, $\mathrm{Y}(\mathrm{t})$ represents individual whale vector position, $\|$ signifies the absolute value and . means element by element multiplication. In addition, $\mathrm{r}$ denotes random vector whose value lies between $[0,1]$ and $a$ is a linearly decreasing element whose value changes from 2 to 0 gradually, during the course of iteration, where $t_{\max }$ denotes the maximum iteration.

$$
\mathrm{a}=2-\left(2 \mathrm{t} / \mathrm{t}_{\max }\right)
$$

The position of each whale is updated corresponding to the location of present optimal individual by adjusting the values of $\mathrm{B}$ and $\mathrm{D}$ it is possible to obtained different locations around current optimal individual. By using the random vector are it is possible to reach any position with in a reliable solution. Using equation 1 any whale can update its position in the neighborhood of the present optimal solution.

\subsection{Bubble Net Attacking}

In the phase of exploitation process, whales swim around prey in a shrinking circle and with a spiral path concurrently.

\subsection{Shrinking Encircling Mechanism}

By decreasing the fluctuation value of $\mathrm{B}$ as shown in the equation 1 shrinking behaviour can be archived. Corresponding to the equation 3 the value of $\mathrm{B}$ can be reduced using a. particularly B is an arbitrarily value whose interval lies between $[-\mathrm{a}, \mathrm{a}]$. to archive new position of an individual then the value of $B$ is set to lie between $[-1,1]$.

\subsection{Spiral Path Mechanism}

In this the distance among whale and the prey is calculated first, and spiral path between whales' current position and the prey is simulated using Helix Shaped Movement of whales, which is represented as follows:

$$
\mathrm{Y}(\mathrm{t}+1)=\mathrm{E}^{\prime} \cdot \mathrm{e}^{\text {bl. }} \cdot \cos (2 \pi \mathrm{l})+\mathrm{Y}(\mathrm{t})
$$

$$
E^{\prime}=|\hat{Y}(t)-Y(t)|
$$

Where $\mathrm{E}^{\prime}$ is the absolute distance among present optimal individual $\hat{\mathrm{Y}}(\mathrm{t})$ and the present individual whale $\mathrm{Y}(\mathrm{t})$ at $\mathrm{t}$ iteration, logarithmic spiral shape is denoted by $b, 1$ represents random number whose value lies between $[-1,1]$ and is element by element multiplication.,

$$
\hat{y}(t+1)=\left\{\begin{array}{c}
\hat{Y}(t)-B \cdot E \quad p<0.5 \\
E^{\prime} \cdot e b l \cdot \cos (2 \pi l)+Y(t) \quad P \geq 0.5
\end{array}\right.
$$

Bubble net behaviour of whale is signified using the above equation.

\subsection{Searching for Prey}

Humpback whales also search the prey in an arbitrary fashion it is achieved by variation of the vector $\mathrm{B}$. When $|\mathrm{B}|<1$, the exploitation is done by means of updating the position of the whale to the current optimal individual when $|\mathrm{B}|\rangle=1$, then position updating is done by choosing the random individual position to achieve global optimum, which is expressed as follows:

$$
\begin{aligned}
& Y(t+1)=Y_{\text {rnd }}(t)-B \cdot E \\
& E=\left|D . Y_{\text {rnd }}(t)-Y(t)\right|
\end{aligned}
$$

Where $Y_{\text {rnd }}(t)$ is the among the present population, the random selected individual position vector of whale.

\section{PROPOSED MODEL FOR CQWOA}

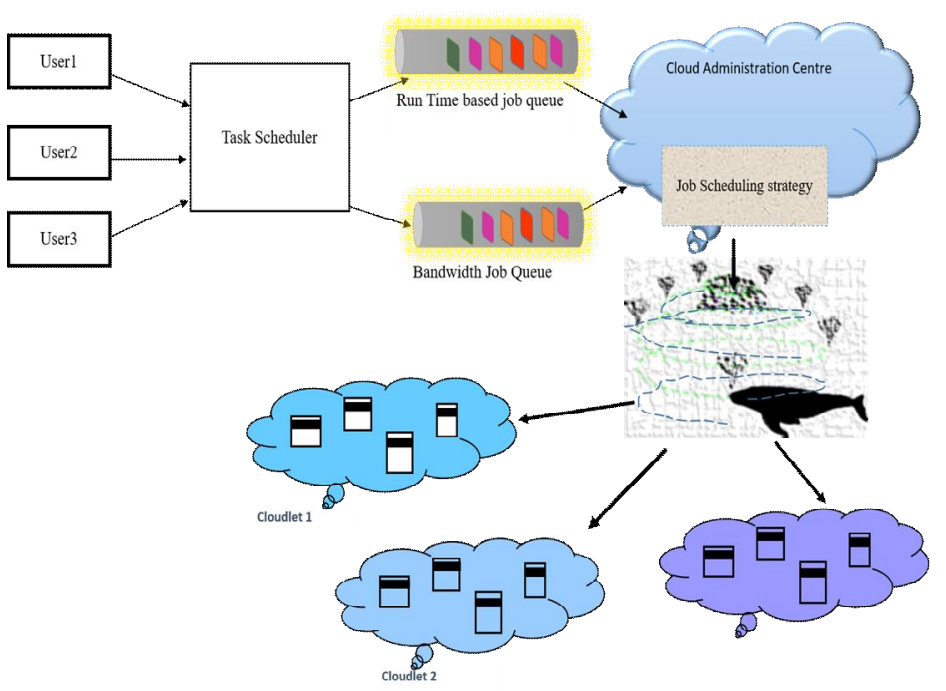

Figure 1: Architecture for Chaos Quantum Whale optimization-based Job Scheduling in Cloud paradigm

In this proposed chaos quantum whale optimization the job scheduling in cloud computing is to handle $\mathrm{N}$ independent job request $\left\{\mathrm{jr}_{1}, \mathrm{jr}_{2}, \mathrm{jr}_{3} \ldots \mathrm{jr}_{\mathrm{n}}\right\}$ on $\mathrm{M}$ resources $=\left\{\mathrm{VM}_{1}, \mathrm{VM}_{2}\right.$ $\left.\mathrm{VM}_{3}, \ldots \mathrm{VM}_{\mathrm{n}}\right\}$. The expected computation runtime time is 
calculated by framing matrix with job task with available virtual machine which is formulated as expected runtime of the task's job request jri on the resource virtual machine $\mathrm{VM}_{\mathrm{j}}$. The overall runtime of job request is obtained by

$$
\mathrm{EJF}_{\mathrm{i}}=\mathrm{S}_{\mathrm{j}}+\min _{j=1, M} \mathrm{EJC}\left(\mathrm{i}_{,} j\right)
$$

Overall execution time of entire the tasks could be stated as trails:

$$
\mathrm{TET}=\max _{i=1 . N} \mathrm{ETC}_{j}
$$

In this work bandwidth consumed of network transmission is also considered in which the total bandwidth of the resource (bw) consumed by all the tasks can be represented as

$$
\mathrm{EBW}=\frac{\theta}{\text { TaskNumber }\left(J R_{m}\right)} \sum_{i=\operatorname{totaltask}(m-1)}^{\operatorname{totalnak}(m)} \ln b w_{m}
$$

Where $\theta$ is a balance constant, and $<\theta \leq 1$

In this proposed model, chaotic quantum whale optimization based balanced job scheduling is done in the cloud computing environment. The job scheduler in cloud administration centre receives users request as illustrated in the figure 1. Once the scheduler receives the request from the user, the job is classified and prioritized based on its expected runtime and bandwidth consumption. The two different lists are constructed, each for runtime and jobs needed bandwidth. Depending on their normalized performance the virtual machine is listed and its information is passed to the job scheduler. Based on expected waiting time and bandwidth to complete a job, the corresponding virtual machine which satisfies the criteria of waiting time and bandwidth is selected from the virtual machine list and assigned for that task. The selection of optimal virtual machine is done using chaotic quantum whale optimization algorithm which imitates the foraging behavior of the humpback whales. The standard whale optimization algorithm suffers from early convergence and premature termination due to local optima results in unfair job scheduling. To overwhelm this problem, the proposed chaotic quantum whale optimization avoids the local optima by moving it to the better state of global optima in an intelligent way and achieves its optimal allocation of job request to the most suitable virtual machines, and lessen the unbalanced job scheduling in cloud environment in a positive way.

\subsection{Initialization of humpback whale's population using chaos strategy}

Chaos theory is an important study area in mathematics which is highly react to initial conditions which is also termed as butterfly effect, if there is any slight modification in one stage of deterministic nonlinear scheme faces consequence in their later stage with huge variation [16]. In standard whale optimization algorithm, the initial population of whales are selected in a random manner. This may fail to select most promising individuals which influence best solution space. The randomness in selecting populations cannot guarantee the uniform distribution in job assignment. And it also results in earlier convergence and suffers from local optima. To overcome this problem, this work introduced chaos mapping which handles the complex nature of WOA with its unpredictable behaviour and mapping strategy. By using chaotic system, it provides possibility of improving uniform distribution of jobs to the appropriate virtual machines. It also avoids earlier convergence by improving the diversity of whale's population and optimal search space by reaching global optimization which avoids earlier convergence of individuals.

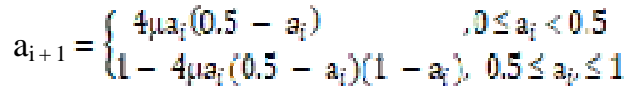

where $3.569946 \leq \mu \leq 4, \mu=4, a_{0}=\operatorname{rand} \in(0,1)$.

\subsection{Quantum Humpback Whales Optimization Algorithm}

In conventional whale optimization, the individuals are defined by their location and position vector, these are the trajectory of whale particle. According to Newtonian mechanism individuals moves along predictable trajectory. But in reality, it is not possible to determine simultaneously distance and position due to the principle of uncertainty. Hence in this work quantum mechanics is adopted to inhibit quantum behaviour in whale optimization algorithm. The quantum model of WOA denotes an individual which has a state depicted by wave function $\psi(x, t)$, instead of using distance and position. Whale's dynamic behaviour is different from standard whale optimization, in which the distance and position of individual cannot computed accurately at same period of time. The probability density function of whale position $x_{i}$ is $(\psi(x, t))^{2}$. the wave function of individual is represented as follows

In this approach, among $\mathrm{W}$ individuals, the position of the $\mathrm{j}^{\text {th }}$ individual $\left(\mathrm{Ind}_{\mathrm{j}}\right)$ in $\mathrm{M}$-dimensional space at $(\mathrm{t}+1)$ iteration is updated as follows

$$
\begin{aligned}
& \mu \mathrm{bst}=\frac{1}{W} \sum_{j=1}^{W} I n d_{j} \\
& \text { Indj }=\tau \operatorname{Ind}_{\mathrm{pbst}}+(1-\tau) \operatorname{Ind}_{\mathrm{gbst}} \\
& \text { If } \operatorname{rnd}()>0.5 \text {, then } \\
& \quad \operatorname{Xpos}_{\mathrm{id}}(\mathrm{t}+1)=\operatorname{Ind}_{\mathrm{id}}-\sigma\left|\mu_{\mathrm{bst}} \mathrm{Xpos}(\mathrm{t})\right| \ln \frac{1}{p} \\
& \text { else } \\
& \quad \operatorname{Xpos}_{\mathrm{id}}(\mathrm{t}+1)=\operatorname{Ind}_{\mathrm{id}}+\sigma\left|\mu_{\mathrm{bst}} \mathrm{Xpos}(\mathrm{t})\right| \ln \frac{\mathbf{1}}{p}
\end{aligned}
$$

Where, $\mu_{\text {bst }}$ is defined as mean best position of personal best position $\operatorname{Ind}_{\mathrm{bst}}$ of the entire individuals in the solution space. The shrinkage extension coefficient is signified as $\sigma$ and $\tau, p$ is two random number all these three values ranges among $[0$, 1]. $\operatorname{Ind}_{\mathrm{bst}}$ is the personal best position of each individual and Indg $_{\text {bst }}$ is the global best position of the whole whale flock. 
G. Kiruthiga et al., International Journal of Advanced Trends in Computer Science and Engineering, 8(4), July- August 2019, 1753 - 1760

\subsection{Algorithm for Chaotic Quantum Whale Optimization Algorithm for job scheduling in Cloud computing}

Input: Jobs (jb1, jb2, jb3, ..., jbn), Virtual machines (VM1, $\mathrm{VM} 2, \ldots, \mathrm{VMk})$

Output: Allocated tasks to the virtual machines in Cloud Computing

\section{BEGIN}

Step 1: Select the initial population of whale's according to the chaotic mapping as shown in the equation (14)

Step 2: Compute the fitness function of each individual involved in searching for resource using the equation (1)

Step 3: Set the current best optimal individual whale as agent $\hat{Y}$ Step 4: While (I < max-iteration)

a. For each search individual

Update E, B, D, r and 1

IF $(E<0.5)$ then

$\operatorname{IF}(|\mathrm{B}|<1)$ then

Update the position of the current search agent using the equation (2)

Elseif $(|\mathrm{B}|>1)$ then

Update the position of the search agent using the quantum mechanism using equation (15) and (16) as follows:

If $\operatorname{rnd}()>0.5$, then

$$
\operatorname{Xpos}_{\mathrm{id}}(\mathrm{t}+1)=\operatorname{Ind}_{\mathrm{id}}-\sigma\left|\mu_{\mathrm{bst}}-\mathrm{Xpos}(\mathrm{t})\right| \ln \frac{1}{p}
$$
else

$$
\mathrm{Xpos}_{\mathrm{id}}(\mathrm{t}+1)=\operatorname{Ind}_{\mathrm{id}}+\sigma\left|\mu_{\mathrm{bst}}-\mathrm{Xpos}(\mathrm{t})\right| \ln \frac{\mathbf{1}}{\mathrm{p}}
$$

\section{End if}

End if

End if

If $(\mathrm{P}>=0.5)$

Update the position of search individual using Equation (6)

End if

End for

If (Any search agent goes beyond the search space and amends it)

Update $\hat{Y}$ using chaotic mapping using equation (14)

$\mathrm{I}=\mathrm{I}+1$

End while

Return $\hat{Y}$

In this proposed algorithm of CQWOA based job scheduling, initially the population of individuals from flock of whale is selecting using the chaotic mapping instead of the random selection which aims at distributing the individuals uniformly to the search space. Here, the solution space is the number of available virtual machines of cloud involved in job processing. The optimal suited virtual machine for a specific job is searched using the chaotic quantum behaviour based whale optimization problem. It determines the currently selected virtual machine and their capacity to handle the complete runtime of the job. The VM which can optimally suit for a completing the task is encircled by the whale by updating their position vector using spiral mechanism or if they fall in local optima then it uses quantum mechanism for discovering such optimal VM resources. Then to reach the concern virtual machine the individuals compare their current best position and the global best position to change their direction towards the optimal search space. The process continues till the jobs are completely scheduled.

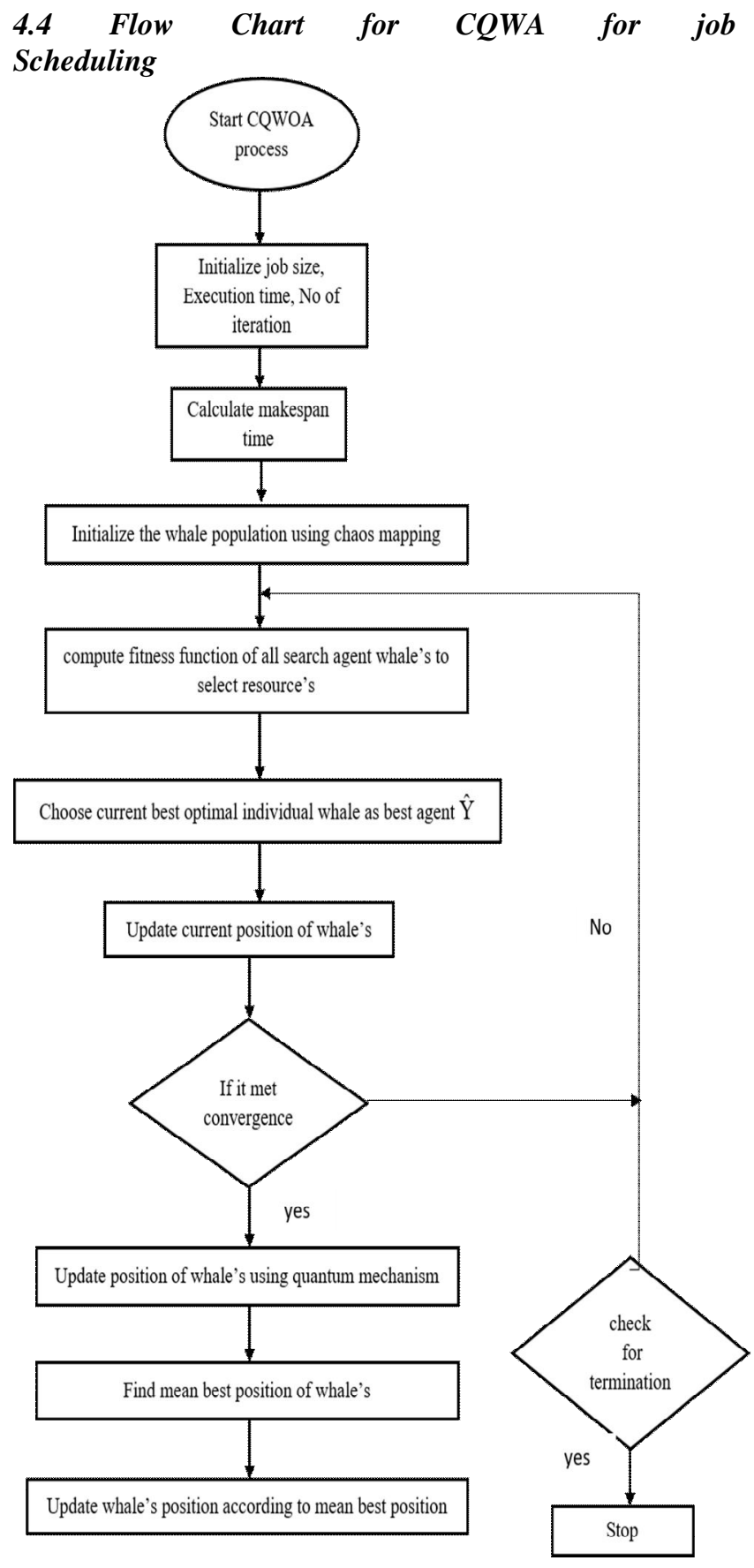




\section{SIMULATION RESULT}

To prove the performance of the proposed CQWOA for solving job scheduling in Cloud Paradigm, the algorithm was coded Cloudsim tool with JAVA and it is run on a Intel Core i5-8250 central processing unit (CPU) with $1.80 \mathrm{GHz}$ frequency, 8 GB random-access memory (RAM), and a Windows 10 Operating System. Performance of CQWOA is compared with Swarm Optimization (PSO), Ant Colony Optimization (ACO), Standard Whale Optimization Algorithm (WOA) scheduling models.

\subsection{Performance Comparison based on Makespan}

Makespan is defines as the total execution time that is needed to execute all independent jobs. The figure 2 shows the performance CQWOA is better compared to PCA, ACO and standard WOA in terms of makespan due to the chaotic and quantum ability of CQWOA.

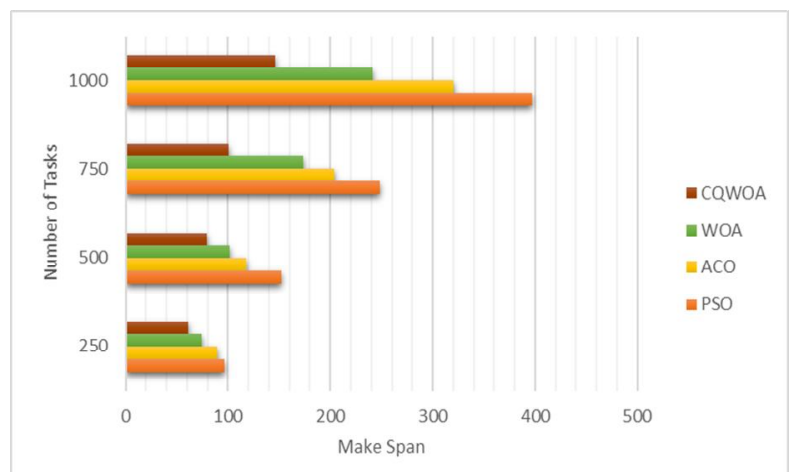

Figure 2: Performance Analysis based on Number of Tasks and Makespan

The chaotic mapping is applied for selecting optimal initial population of humpback whales to and to uniformly distributing the individuals in search space to discover more appropriate virtual machine to complete the selected job. In real time both the direction and velocity cannot be determined simultaneously, so that CQWOA applies quantum mechanism which updates the location of other whales by finding mean value of the best individuals to avoid local optima and works in a parallel manner. These reasons increase the makespan while using CQWOA job scheduling.

\subsection{Performance analysis based on cost consumption}

The Cost estimation defines the execution cost of tasks execution on a specific virtual machine in cloud computing environment. Cost is computed based on jobs length, data transfer tasks and the storage capacity.

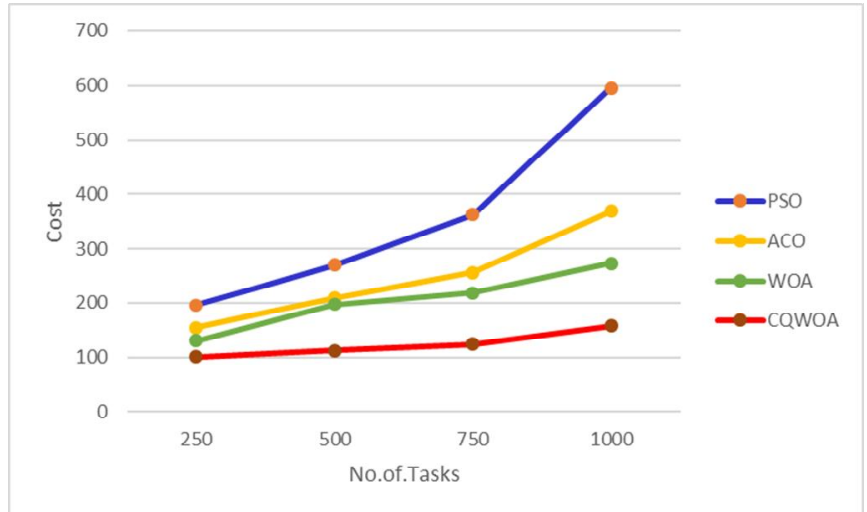

Figure 3: Performance comparison based on cost versus number of tasks

When there are a greater number of jobs are waiting for processing, then using PSO, ACO and standard WOA easily falls under local optima and earlier convergence because global optimal solution becomes complex for these scheduling schemes. But while using CQWOA earlier convergence and the searching direction is greatly controlled by adapting both chaotic and quantum behaviour of the humpback whales which greatly achieves the global optimization by choosing new set of population using chaotic mapping when the remaining whales which involved in resource finding fails to choose optimal virtual machines to process jobs in queue. Thus, from the figure 3 it is observed that the cost is highly reduced while using the proposed CQWOA while comparing with other three models. The standard WOA also falls under earlier convergences which leads to local optima, they search only nearby virtual machines and doesn't have the capability to uniformly distribute the individual whales as search agent.

\subsection{Performance Analysis based on Degree of Imbalance (DOI)}

The degree of imbalance is defined as the measure of imbalance among VMs to perform job scheduling in cloud environment. It is computed based on the maximum executing time $\left(E T_{\max }\right)$, minimum execution time $\left(E T_{\min }\right)$ and capability average of execution time $\left(E T_{A v g}\right)$ of virtual machines. The behaviour of degree of imbalance is represented as

$$
D O I=\frac{E T_{\max }-E T_{\text {mine }}}{E T_{\text {Avg }}}
$$




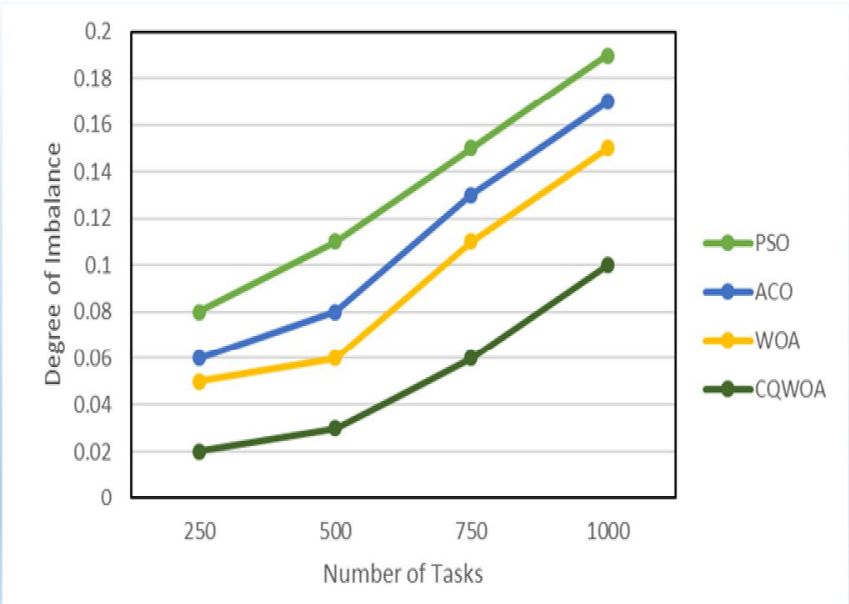

Figure 4: Degree of imbalance

Figure 4 shows the simulation result based on degree of imbalance which occurs due to overloading of jobs to a specific machine while other machines are idle. Four different job scheduling schemes performance are compared with varied number of tasks like $250 \mathrm{~m} \mathrm{500m} 750$ and 1000 on 30 virtual machines. It is obviously proved that the performance of CQWOA is more efficient in balancing the job scheduling by uniformly distributing the jobs to the appropriate virtual machines with the intelligence of quantum mechanism which determines the mean best virtual machines among the other individual best VMs in terms of factors such as length of jobs, priority of execution jobs and the bandwidth of virtual machine. Instead of randomness in choosing whales as search agent this algorithm uses mapping for selecting more intelligent whales, thus it leads to less degree of imbalance while comparing the other three algorithms WOA, PSO and ACO.

\subsection{Performance comparison based on Energy Consumption}

While performing analysis based on the utilization of the CPU and resources which are main factors of energy consumption by a job.

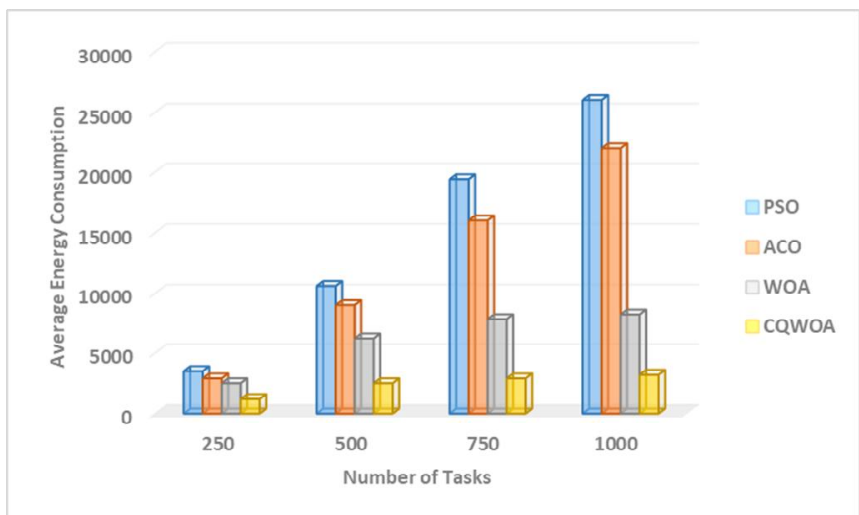

Figure 5: Performance comparison based on Energy Consumption
Energy is consumed high if a processor of virtual machine is not employed and also due to heavy request of resources is also another important factor. Consumption of energy relies on overall execution time of virtual machine. CQWOA consumes less energy because it has low makespan. Selection of optimal virtual machines which can accomplish the job scheduling tasks. The performance of energy consumption by four different scheduling schemes is shown in the figure 5 . The exploration, exploitation and local optimal avoidance are greatly enriched using the chaotic quantum behavior of the CQWOA.

\subsection{Performance Comparison based on Resource Utilization}

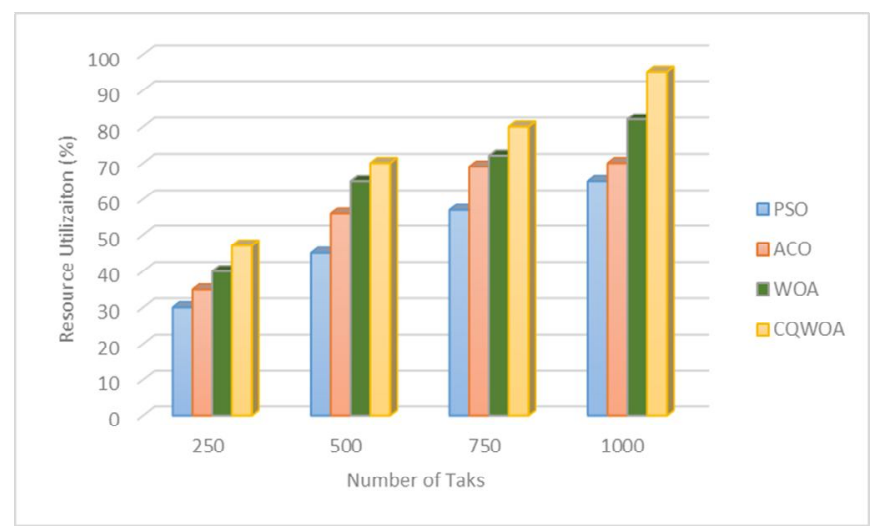

Figure 6: Performance comparison based on Resource Utilization

From the figure 6 it is observed that utilization of resources are greatly increased by using the intelligence of CQWOA to receive the maximum benefit from the cloud computing service providers by renting limited resources to the clients with increased make span by selecting the optimal virtual machines which will complete the task of allocated job with even distribution of work load and thus avoids the overloading problem more positively while comparing the standard WOA, ACO and PSO scheduling schemes.

\section{CONCLUSION}

Even though there are many metaheuristic search methods are available for job scheduling scheme, this paper introduces a novel Chaotic Quantum Whale Optimization algorithm whose aim is to optimize the process of job scheduling in cloud computing. The best virtual machine to complete the task of an arrived job is discovered by the potential search agent of humpback whales which are selected using chaotic mapping strategy. And in addition, all the metaheuristic algorithms fall into earlier convergences local optima and are the toughest challenge, which is greatly handled using quantum mechanism which achieves global optima more easily and enhances the parallel processing in cloud environment more significantly. From the simulation outcomes it is observed clearly that the developed CQWOA achieves superior results while comparing other existing 
models like PSO, ACO and conventional WOA in terms of energy consumption, resource utilization, makespan, cost and degree of imbalance. Thus, chaotic quantum nature of Whale optimization performs a highly balanced and effective utilization of resources in cloud computing to achieve job scheduling more precisely.

\section{REFERENCES}

1. R. Buyya, C.S. Yeo, S. Venugopal, I. Brandic, Cloud computing and emerging IT platforms: Vision, Hype, and reality for delivering computing as the 5th utility, Future Gen. Comput. Syst. 25 (6) (2009) 599-616.

https://doi.org/10.1016/j.future.2008.12.001

2. W. Chen, G. Xie, R. Li, Y. Bai, C. Fan, K. Li, Efficient task scheduling for budget constrained parallel applications on heterogeneous cloud computing systems, Future Gen. Comput. Syst 74 (2017) 1- 11. https://doi.org/10.1016/j.future.2017.03.008

3. S. Bitam, "Bees Life Algorithm for job scheduling in cloud computing," in International Conference on Computing and Information Technology. ICCIT, 2012, pp. 186-191.

4. A. G. Delavar, M. Javanmard, M. B. Shabestari, and M. K. Talebi, "RSDC (reliable scheduling distributed in cloud computing)," International Journal of Computer Science, Engineering and Applications (IJCSEA) Vol, vol. 2, 2012.

https://doi.org/10.5121/ijcsea.2012.2301

5. A. Verma and S. Kaushal, "Deadline and budget distribution-based cost-time optimization workflow scheduling algorithm for cloud," in of the IJCA on International Conference on Recent Advances and Future Trends in Information Technology (iRAFIT'12), 2012, pp. 1-4.

6. L. Wang and L. Ai, "Task Scheduling Policy Based on Ant Colony Optimization in Cloud Computing Environment," in LISS 2012, ed: Springer, 2013, pp. 953-957.

https://doi.org/10.1007/978-3-642-32054-5_133

7. Demyana Izzat Esa, Adil Yousif, Scheduling Jobs on Cloud Computing using Firefly Algorithm International Journal of Grid and Distributed Computing Vol. 9, No. 7 (2016), pp.149-158

https://doi.org/10.14257/ijgdc.2016.9.7.16

8. Jun Xue $\mathrm{S}, \mathrm{Wu} \mathrm{W}$, "Scheduling Workflow in Cloud Computing Based on Hybrid Particle Swarm Algorithm," TELKOMNIKA Indonesian Journal of Electrical Engineering (10:7); 2012, pp. 1560-1566. https://doi.org/10.11591/telkomnika.v10i7.1452

9. Guo L,Zhao S,Shen S, Jiang C, "Task Scheduling Optimization in Cloud Computing Based on Heuristic Algorithm," Journal of Networks (7);2012, pp. 547-553. https://doi.org/10.4304/jnw.7.3.547-553

10. Pandey S, Wu L, Guru S, Buyya R,"A Particle Swarm Optimization-Based Heuristic for Scheduling Workflow Applications in Cloud Computing Environments",24th
IEEE International Conference on Advanced Information Networking and Applications (AINA);2010. https://doi.org/10.1109/AINA.2010.31

11. Varalakshmi P, Ramaswamy A, Balasubramanian A,Vijaykumar P,"An Optimal Workflow Based Scheduling and Resource Allocation in Cloud",Advances in Computing and Communications, First International Conference, ACC;2011, pp. 411-420. https://doi.org/10.1007/978-3-642-22709-7_41

12. Zhong H, Tao K, Zhang X, "An Approach to Optimized Resource Scheduling Algorithm for Open-Source Cloud Systems", in Fifth Annual China Grid Conference;2010. https://doi.org/10.1109/ChinaGrid.2010.37

13. Selvarani S, Sadhasivam G, "Improved cost-based algorithm for task scheduling in cloud computing", IEEE International Conference on Computational Intelligence and Computing Research (ICCIC); 2010.

14. Mirjalili, S, \& Lewis, A., The whale optimization algorithm. Advances in Engineering Software, 95, 51-67, 2016.

15. Reddy, P. D. P., Reddy, V. V., \& Manohar, T. G. Whale optimization algorithm for optimal sizing of renewable resources for loss reduction in distribution systems. Renewables: Wind, Water, and Solar, 4(1), 3, (2017).

16. Fan J. L, Zhang, X. F, Piecewise logistic chaotic map and its performance analysis. Acta Electronica Sinica, 37(4), 720-725, 2009.

17. Ramezani, F., Jie, L., and Hussain, K. (2014) "Task-Based System Load Balancing in Cloud Computing Using Particle Swarm Optimization", International Journal of Parallel Programming, Vol. 42, No. 5, pp. 739-754.

18. Pacinia, E, Mateosb, C, Garino, C. (2015). "Balancing throughput and response time in online scientific Clouds via Ant Colony Optimization", Advances in Engineering Software, Vol. 84, No. 1, pp. 31-47.

19. Karnam Sreenu, M. Sreelatha, W-Scheduler: whale optimization for task scheduling in cloud computing Cluster Comput, pp 1-12, 2017

20. Jhila Nasiri, Farzin Modarres Khiyabani, A whale optimization algorithm (WOA) approach for clustering, Cogent Mathematics \& Statistics (2018), 5: 1483565

21. B. Madhuravani, N.Chandra Sekhar Reddy, K.Sai Prasad, B.Dhanalaxmi, V. Uma Maheswari, "Strong and Secure Mechanism for Data Storage in Cloud Environment", International Journal of Advanced Trends in Computer Science and Engineering, Volume 8, No.1.3, 2019. https://doi.org/10.30534/ijatcse/2019/0681.32019

22. Muhammad Azizi Mohd Ariffin, Khadijah Ab Rahman, Mohamed Yusof Darus, Norkhushaini Awang, Zolidah Kasiran, "Data Leakage Detection in Cloud Computing Platform", International Journal of Advanced Trends in Computer Science and Engineering, Volume 8, No.1.3, 2019. https://doi.org/10.30534/ijatcse/2019/7081.32019 